\title{
ERRATA
}

\section{Erratum: Hexagonal optical structures in photorefractive crystals with a feedback mirror [JETP 86, 614-627 (March 1998)]}

P. M. Lushnikov

L. D. Landau Institute of Theoretical Physics, Russian Academy of Sciences, 117334 Moscow, Russia Zh. Éksp. Teor. Fiz. 115, 378 (January 1999)

[S1063-7761(99)02901-7]

1. The unnumbered equation following Eq. (42) on page 623 should read

$$
\delta \Psi_{k}=\sum_{\mathbf{k}_{1}+\mathbf{k}_{2}=\mathbf{k}} A_{k_{1}} A_{k_{2}} \psi_{k_{1}} \psi_{k_{2}}
$$

2. At the beginning of the second paragraph on page 623 "Substituting Eqs. (42) and (43) into ...." should be replaced by "Substituting Eq. (42) into ...."

3. Equation (43) on page 623 should be replaced by

$$
\begin{aligned}
\frac{\partial A_{k}}{\partial t}= & \nu_{k} A_{k}+\frac{U}{2} \sum_{\mathbf{k}_{1}+\mathbf{k}_{2}=\mathbf{k}} A_{k_{1}} A_{k_{2}}-\sum_{\mathbf{k}_{1}+\mathbf{k}_{2}+\mathbf{k}_{3}=\mathbf{k}}\left\{\left[-\left\langle\psi_{\mathbf{k}}^{c(0)} \mid \eta\left(\psi_{k_{1}}^{(0)}, \psi_{k_{2}}^{(0)} \psi_{k_{3}}^{(0)}\right)+\eta\left(\psi_{k_{2}}^{(0)} \psi_{k_{3}}^{(0)}, \psi_{k_{1}}^{(0)}\right)\right\rangle\right.\right. \\
& \left.\left.-\left\langle\psi_{k}^{c(0)} \mid \Theta\left(\psi_{k_{1}}^{(0)}, \psi_{k_{2}}^{(0)}, \psi_{k_{3}}^{(0)}\right)\right\rangle\right] /\left\langle\psi_{k}^{c(0)} \mid \mathbf{J} \psi_{k}^{(0)}\right\rangle\right\} A_{k_{1}} A_{k_{2}} A_{k_{3}},
\end{aligned}
$$

Translated by M. E. Alferieff 